Research Paper

\title{
Ethnodrama: An Innovative Knowledge Translation Tool in the Management of Lymphedema
}

\author{
Shahid Ahmed ${ }^{\circledR}$, Elizabeth Quinlan², Linda McMullen³, Roanne Thomas ${ }^{4}$, Pam Fichtner ${ }^{5}$, Janice Block ${ }^{6}$ \\ 1. Saskatoon Cancer Center, Department of Oncology, University of Saskatchewan, Saskatoon, SK, Canada; \\ 2. Department of Sociology, University of Saskatchewan, Saskatoon, SK, Canada; \\ 3. Department of Psychology, University of Saskatchewan, Saskatoon, SK, Canada; \\ 4. School of Rehabilitation Sciences, University of Ottawa, Ottawa, ON, Canada; \\ 5. Sephira Healing, Saskatoon, SK, Canada; \\ 6. Department of Physical Therapy, Royal University Hospital, Saskatoon, Canada.
}

$\triangle$ Corresponding author: Shahid Ahmed, MD, FRCPC, FACP, Clinical Professor of Medicine. Saskatoon Cancer Center, 20 Campus Drive, University of Saskatchewan, Saskatoon, SK, Canada, S7N4H4. Phone: (306) 655-2710. Fax: (306) 655-0633. E-mail:

shahid.ahmed@saskcancer.ca

() 2015 Ivyspring International Publisher. Reproduction is permitted for personal, noncommercial use, provided that the article is in whole, unmodified, and properly cited. See http:/ /ivyspring.com/terms for terms and conditions.

Received: 2015.03.29; Accepted: 2015.06.23; Published: 2015.07.16

\begin{abstract}
Background: Lymphedema can cause significant physical impairment and quality-of-life issues. Yet there is a gap in knowledge about lymphedema among breast cancer survivors (BCS), and health care professionals (HCP). Ethnodrama is an innovative knowledge translation strategy that uses theatrical performances for dissemination of research results. We evaluated the impact of live ethnodrama on HCP' and BCS' awareness and attitudes in relation to impact of lymphedema on BCS' lives.

Methods: Ethnodrama performances were developed by script writers and a theatre director in collaboration with the investigators and BCS using data from published research and pre-performances workshops. Six interactive live performances were given to audiences of BCS, $\mathrm{HCP}$, and community members in four cities across Canada. After watching these live performances, members of the audiences were asked to complete a paper-based questionnaire regarding their knowledge of lymphedema, and their attitudes and practices toward lymphedema.

Results: Of 238 audience members who participated in the survey, 55 (23\%) were BCS and 85 (37.5\%) were HCP. Most members rated the performances as very effective in changing their (84\%) or other people's (93\%) understanding of lymphedema; $96 \%$ reported being motivated to seek additional information on lymphedema, and $72 \%$ of $\mathrm{HCP}$ anticipated changes in their practices related to lymphedema screening. Overall no significant differences were noted in responses to ethnodrama between BCS and HCP. Open-ended responses were supportive of the findings from the closed-ended questions.

Conclusions: Our results indicate that ethnodrama performances effectively convey information and positively affecting changes in HCP' and BCS' attitudes toward lymphedema.

Key words: Ethnodrama, lymphedema, knowledge translation, arm morbidities, breast cancer, survivorship, interactive theatre, attitude, health care professionals
\end{abstract}

\section{Introduction}

The arm morbidities of pain, impaired range of motion, and lymphedema are common among breast cancer survivors following breast cancer treatment $[1,2]$. Among them lymphedema is the most debili- tating complication of treatment of breast cancer [1-4]. The prevalence of lymphedema in breast cancer survivors varies from 0 to more than 50 percent. The risk of lymphedema is as high as 59 percent in women 
who are treated with both axillary lymph node dissection and axillary radiotherapy [3]. Although in most clinical settings sentinel lymph node biopsy (SLNB) has replaced complete axillary lymph node dissection as the standard staging procedure, the risk of lymphedema is not insignificant [4-6]. A prospective trial demonstrated that 5 percent of women with clinically node-negative breast cancer who underwent SLNB alone developed lymphedema at a median follow up period of five years [5]. Others have reported the risk of lymphedema as 3.5 to 7 percent following SLNB $[1,6,7]$.

There is growing evidence that women with lymphedema have significant impairment in their quality of life and physical functioning [8-15]. In our longitudinal parent study, 745 women with early stage breast cancer were enrolled at various sites across Canada and were followed for five years. The study revealed that lymphedema increases for the average survivor up to 3 years post-surgery and lymphedema and other arm morbidities have a significant impact on various aspects of survivors' lives, such as work, leisure, and relationships [12-16]. Other investigators have also reported that breast cancer survivors with lymphedema or arm symptoms have lower physical and mental health-related quality-of-life $[8,9]$.

Although interventions aimed at improving arm function such as lymphedema education, use of light arm weights with exercises, light compression sleeves during vigorous arm use, and regular breathing exercises have been used to reduce the incidence and severity of lymphedema following breast cancer treatment, there is still a general lack of awareness of lymphedema and its impacts among breast cancer survivors and health care professionals [2,9,17-19]. Most physicians report that they do not routinely counsel or provide written information on lymphedema prevention to their patients, and the extent to which breast cancer survivors' daily living is affected by the condition is not always recognized [2, 19].These findings point to the need for interventions to improve awareness of lymphedema. Early detection and prevention of this treatment-related morbidity can be more effective than late identification and intervention. Question remains, however, the best ways of providing information to health professionals [20].

Arts-based methods are a unique way of creating new knowledge and translating the findings [21-23]. A recent review outlines the many possibilities and connections between the arts and health research [24]. Ethnodrama is an innovative knowledge translation strategy and a dissemination tool that uses theatrical performances based on research findings to dissemi- nate research results to a variety of stakeholders $[21,23]$.

The study upon which this article is based involved creating, presenting, and exploring the impact of arm morbidity ethnodrama performances on knowledge translation among breast cancer survivors and professional health care members [25]. The three aims of the study were (1) to foster knowledge of impact of arm morbidity on breast cancer survivors' work, leisure, and relationships by using interactive ethnodrama; (2) to explore the capacity of interactive performances to change attitudes and practices related to arm morbidity of breast cancer survivors and health care professionals; and (3) to investigate interactive ethnodrama as a potential psychosocial intervention for breast cancer survivors.

In the present report we focus on the second aim and evaluate the impact of live ethnodrama on health care professionals' and survivors' awareness and attitudes in relation to effects of lymphedema on breast cancer survivors' lives using post-performance questionnaires.

\section{Methods}

\section{Development of Ethnodrama}

The study was approved by the University of Saskatchewan' Ethics Board. In phase I, the ethnodrama script was developed using findings from the parent project combined with data collected with breast cancer survivors in two day-long workshops in which creative arts were used to solicit participants' experiences of living with lymphedema The workshops involved breath work, yoga, collage or writing (or both), discussions, surveys, as well as found-art installations. Seven breast cancer survivors with lymphedema participated. Three script writers and a theatre director worked with the investigators and the participants to develop the production. The 25- minute performance follows a readers' theatre model with four characters performed by the workshop participants and the director filling in when necessary. The actors read from the script, costumes and props are minimal, and the backdrop features photographs of the participants' collages and installations. Six interactive ethnodrama performances conducted in four cities across Canada over a period of 14 months: two performances each in Saskatoon, SK and Toronto, ON, one each in Regina, SK and St. John, NB. One of the performances (The New Normal: Living with Lymphedema) is available on https://www.youtube. com/channel/UCewEVF6CcTlEKYILNcc8FWQ

\section{Post-Performance Surveys}

Members of the audiences were surveyed after each performance. Follow-up in-depth interviews 
were conducted via telephone with a sub-group of audience members. The questionnaire was organized into the following sections: demographic characteristics, participants' knowledge of lymphedema, and participants' attitudes and practice toward lymphedema in relation to their viewing of the ethnodrama. The surveys also included open-ended questions.

Descriptive statistics were used to generate frequencies for each relevant item using SPSS version 22 (SPSS Inc, Chicago, IL). Results are presented in tabular format using absolute values and percentages using a 5-point Likert-style scale. Fisher Exact test was performed for categorical variables. A two-tailed $p$ value of $<0.05$ was considered statistically significant. Open-ended responses were evaluated independently.

\section{Results}

\section{Demographic Characteristics of Respondents}

The total number of audience members who participated in the post-performance survey was 238, with a mean number of 40 (range 28-64) audience members per performance. The mean age of respondents was 46 years (standard deviation, $15 \mathrm{yrs}$ ) and male to female ratio was 1:4.8. Of the 238 respondents, $55(23 \%)$ were breast cancer survivors and $85(37.5 \%)$ were health care professionals; the remaining participants were survivors' family members and other interested community members such as teachers, educators, and students. More than 90 percent of the respondents either had completed post-secondary education or were university students. The health care professionals included, but were not limited to, medical doctors, registered nurses, physiotherapists, occupational therapists, and allied health providers.

\section{Summary of Findings}

Overall approximately 84 percent of the respondents rated the performances as very effective or extremely effective in changing their understanding of arm morbidities following breast cancer surgery (Table 1A): 80.4 percent of health care professionals; 75 percent of breast cancer survivors, and 92 percent community members $(p=0.52)$. Overall 2.7 percent of respondents judged the performances as not effective at all in changing their understanding of arm morbidities following breast cancer treatment compared with 97.3 percent respondents who found them effective $(\mathrm{p}<0.001)$ [Figure 1A]. About 93 percent of respondents (90.5 percent of health care professionals versus 90.9 percent of breast cancer survivors) rated the performances as very effective or extremely effective in changing other people's understanding of arm problems after breast cancer surgery. No respondents judged the performances as not effective at all in changing other people's understanding of arm problems (Figure 1B). Following the performances, 96.3 percent respondents were motivated to seek out more information about arm problems compared with 3.7 percent respondents who were not at all motivated $(p<0.001)$. Of all respondents approximately 87 percent (95.0 percent of health care professionals versus 86.2 percent of breast cancer survivors, $\mathrm{p}=0.10$ ) reported being moderately to extremely motivated to seek additional information on lymphedema. Of 51 breast cancer survivors, $6(11.7 \%)$ reported not being motivated to seek additional information compared to one $(1.2 \%)$ of 81 health care professionals $(p=0.01)$. Otherwise no significant differences between breast cancer survivors and health care professionals were noted in their responses to the ethnodrama performances (Table 1A).
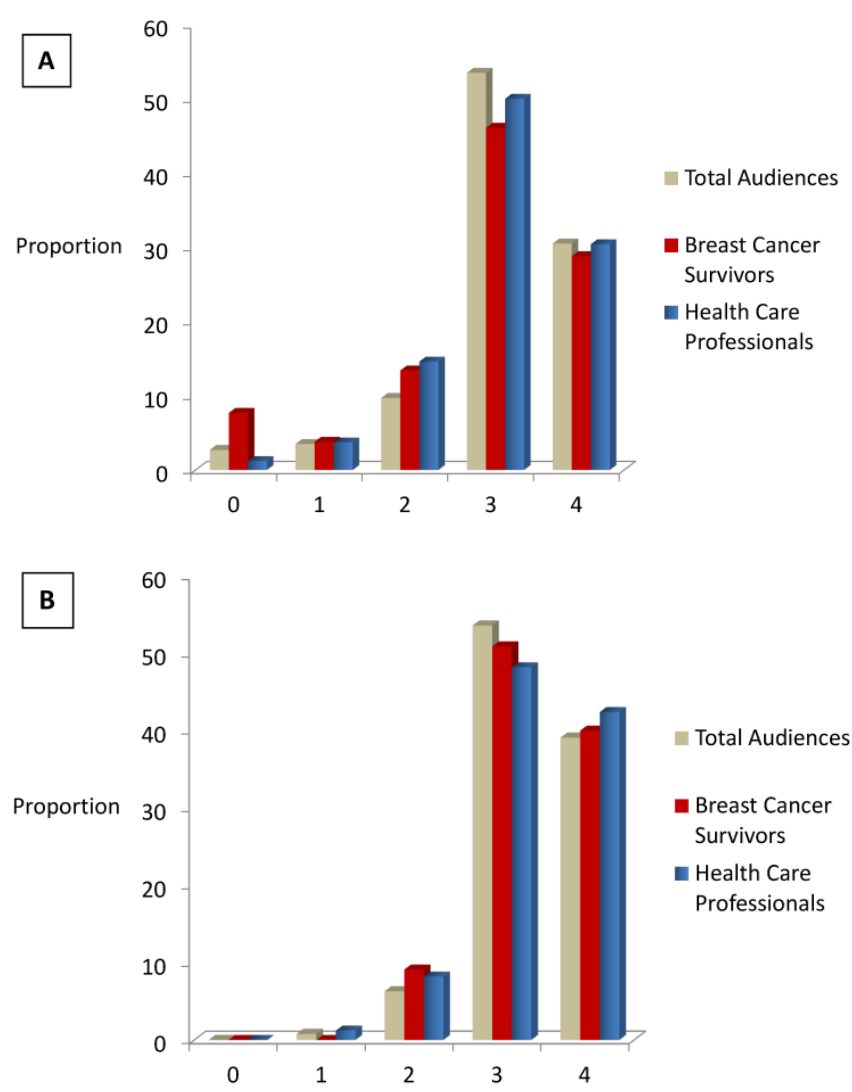

Figure 1: Perceptions of audiences in relation to effectiveness of the performance in changing $(\mathbf{A})$ their and $(\mathbf{B})$ other people's understanding of arm problems after breast cancer surgery. 0 to 4 five points scale with 0 not at all effective and 4 is extremely effective.

After observing the performances, 76 percent of the respondents who were breast cancer survivors reported interest in discussing arm morbidity issues with their health care professionals; about 60 percent of them anticipated asking their providers about a 
referral to physio- and occupational therapists (Table 1B). Seventy-two percent of health care professionals reported anticipated changes in their practice regarding screening of arm morbidities. Comments from open-ended questions supported or provided further explanation for the findings from the closed-ended questions (Tables 2 and 3). For example, one of the breast cancer survivors commented, "I was totally ignorant - now I know something!" A health care professional expressed, "I learned about all the emotional stress the patients encounter, their outlook, [and the] lack of support from the healthcare system".

\section{Discussion}

Although recent progress in breast cancer treatment has resulted in a significant increase in the number of survivors, many of them face long-term unintended adverse effects of the treatments that have saved their lives [26]. Cancer follow-up care tends to focus on the primary disease, rather than on the impact of the illness or treatment-related disabilities. Furthermore, there is a knowledge gap regarding long-term treatment-related physical and emotional disabilities among breast cancer survivors and health care providers $[2,9,19,27]$.

Table 1A: Summary of the findings about knowledge and attitudes of the participants in response to interactive ethnodrama.

\begin{tabular}{|c|c|c|c|c|}
\hline Grade & $\begin{array}{l}\text { Total Participants } \\
\mathrm{N}=238(\%)\end{array}$ & $\begin{array}{l}\text { Breast Cancer Survivors } \\
\mathrm{N}=55(\%)\end{array}$ & $\begin{array}{l}\text { Health Care Professionals } \\
\mathrm{N}=85(\%)\end{array}$ & $\mathrm{P}$ values \\
\hline \multicolumn{5}{|c|}{ How effective was the performance in changing your understanding of arm problems after breast cancer surgery? $\Phi$} \\
\hline Not at all effective & $6(2.7)$ & $4(7.7)$ & $1(1.2)$ & 0.07 \\
\hline Slightly effective & $8(3.5)$ & $2(3.8)$ & $3(3.7)$ & 1.0 \\
\hline Moderately effective & $22(9.7)$ & $7(13.4)$ & $12(14.6)$ & 1.0 \\
\hline Very effective & $121(53.5)$ & $24(46.1)$ & $41(50)$ & 0.72 \\
\hline Extremely effective & $69(30.5)$ & $15(28.8)$ & $25(30.4)$ & 1.0 \\
\hline \multicolumn{5}{|c|}{ How effective might the performance be in changing other people's understanding of arm problems after breast cancer surgery? } \\
\hline Not at all effective & 0 & 0 & 0 & \\
\hline Slightly effective & $2(0.8)$ & 0 & $1(1.2)$ & 1 \\
\hline Moderately effective & $15(6.3)$ & $5(9.1)$ & $7(8.2)$ & 1 \\
\hline Very effective & $126(53.6)$ & $28(50.9)$ & $41(48.2)$ & 0.86 \\
\hline Extremely effective & $92(39.1)$ & $22(40)$ & $36(42.4)$ & 0.86 \\
\hline \multicolumn{5}{|c|}{ How motivated are you to seek out more information about arm problems after breast cancer surgery as a result of the performance?TT } \\
\hline Not at all motivated & $8(3.7)$ & $6(11.7)$ & $1(1.2)$ & 0.01 \\
\hline Slightly motivated & $19(8.8)$ & $1(1.2)$ & $3(3.7)$ & 1 \\
\hline Moderately motivated & $63(29.4)$ & $11(21.5)$ & $23(28.3)$ & 0.42 \\
\hline Very motivated & $88(41.1)$ & $24(47.1)$ & $37(45.6)$ & 1 \\
\hline Extremely motivated & $36(16.8)$ & $9(17.6)$ & $17(20.9)$ & 0.82 \\
\hline
\end{tabular}

TOf 238 audiences, 12 did not respond to this question ( three breast cancer survivors and three health care professionals did not respond); TT Three audiences did not respond; T†T Twenty four audiences did not reply to this question (Four breast cancer survivors and health care professionals did not respond).

Table 1B: Summary of the findings about knowledge and attitudes of the participants in response to interactive ethnodrama.

\begin{tabular}{|l|l|l|}
\hline Breast Cancer Survivors & Yes & No \\
\hline $\mathrm{N}=55(\%)$ & $42(76.4)$ & $13(23.6)$ \\
\hline $\begin{array}{l}\text { As a result of the performance, will you raise the topic of arm problems after surgery with your main health } \\
\text { care provider? }\end{array}$ & $28(59.6)$ & \\
\hline As a result of the performance, do you anticipate asking for referral to other providers? & Yes & No \\
\hline Health Care Professionals & $61(71.8)$ & $24(28.2)$ \\
\hline $\mathrm{N}=85(\%)$ & $\begin{array}{l}\text { As a result of the performance, do you anticipate changing your practice regarding screening for arm prob- } \\
\text { lems among your patients who have had breast cancer surgery? }\end{array}$ & \\
\hline
\end{tabular}

T eight (14.5\%) breast cancer survivors did not reply to this question.

Table 2: Selected comments by the breast cancer survivors from open-ended responses.

Comments by breast cancer survivors following ethnodrama performances

I was totally ignorant - now I know something!

It was good people can talk about feelings.

I have better understanding of the emotional side of all your experiences; many experiences and many emotions.

Identifying and understanding that I am not alone.

This reinforced my ideas that I'm not the only one who had problems.

Effective because it's my story as well.

Already have lymphedema - understand your feelings.

The performance made me realize how many women are really affected by lymphedema - I am not alone!

Knowing that I can make a difference as support if not a difference in lymphedema management.

Lymphedema is real - not a figment of imagination.

I could feel them. I could see them. 
Table 3: Selected comments by the health care professionals from open-ended responses.

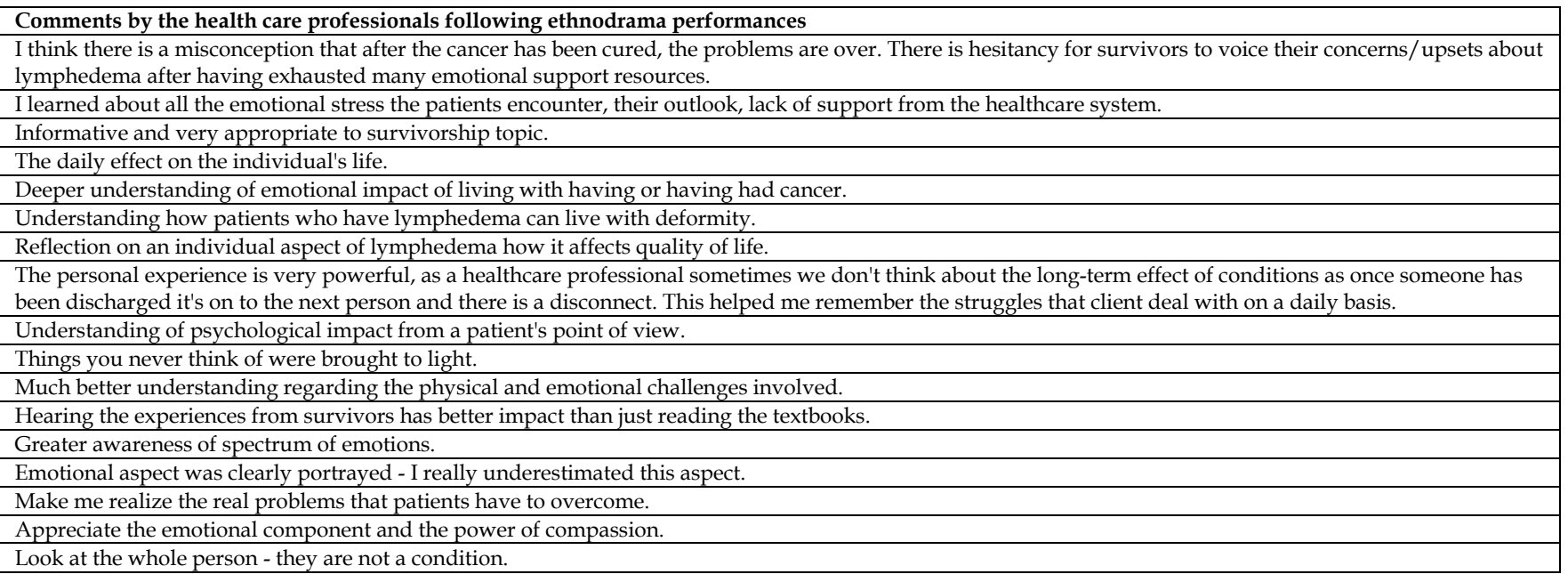

Traditional strategies to disseminate new knowledge from health research have largely focused on the publication of research results in academic journals. Ethnodrama, a dramatic performance of the lived experience, is an innovative way to convey knowledge about treatment-related disabilities in cancer survivors. Our results indicate that ethnodrama can have a positive effect on health care professionals' and breast cancer survivors' awareness, attitudes, and practices. The majority of our respondents rated the performances as very effective in changing their and other people's understanding of arm problems after breast cancer surgery.

Most health care professionals who completed our questionnaires reported being persuaded to seek additional information about lymphedema when they were asked about their education-seeking behaviors. Moreover, many reported anticipating potential changes in their practice regarding screening for lymphedema in breast cancer survivors.

With respect to patients, international best practice guidelines indicate that knowledge and self-care are important aspects of the management of arm morbidity symptoms related to breast cancer survivorship. In this regard, the interactive performances appear to be potentially useful for addressing gaps in knowledge among the breast cancer survivors. Most breast cancer survivors reported being interested in seeking additional information about lymphedema and discussing it further with their health care providers. Of note, six breast cancer survivors, compared with one health care provider, reported not being motivated to look for more information on lymphedema. It is conceivable that these survivors were already suffering from arm morbidities and were well educated. Apart from this finding, our results did not reveal significant differences in the per- ceived effects of the ethnodrama on attitudes of health care professionals compared to those of breast cancer survivors. In addition, community members have similar attitudes to ethnodrama performances. More than 90 percent community members rated the performances as very effective or extremely effective in changing their or other people's understanding of lymphedema following breast cancer treatment.

The open-ended responses to the performances signify the engagement and critical reflection of members of the audiences. Several breast cancer survivors felt that their stories and experiences were voiced and indicated that this reduced their feelings of isolation: they felt that they are not alone in dealing with the post-treatment hardship. Likewise, following the performances, many health care professionals were appreciative of their improved insights into the emotional aspect of arm morbidities and the potential offered by a holistic approach to cancer care -- i.e., a focus on the whole person, rather than just on the disease - which is consistent with contemporary emphases on person-centered care.

The standard treatment for lymphedema is not defined; nevertheless, early diagnosis and prevention are vital to minimize long-term impacts $[2,10,17]$. Improvements in care, including screening of arm problems and timely referrals to appropriate services, can be achieved through improved knowledge transfer to health care professionals and breast cancer survivors. In a conventional model of knowledge translation, knowledge is understood as a product (information) to be created, transferred, and applied in a linear manner, but the demand for novel strategies to bridge the theory-to-practice gap in the health care is increasingly recognized [28-30]. An emerging model of knowledge translation conceives of the process as being dynamic, iterative, and situated within a com- 
plex system of interactions between researchers and stakeholders [31,32]. Theatre is a unique and powerful medium to access new knowledge by invoking intuitive, affective and cognitive responses [21]. In interactive ethnodrama, also known as participatory or applied theatre, scenarios are developed by a core group of participants to reflect the underlying stories of their everyday lived experience. Only a minimum of sets, props, and costumes are used and no previous acting training is required by participants. Consequently, ethnodrama can be a very functional medium for conveying health research findings, with significant potential to change attitudes and practices. Moreover, ethnodrama can be a valuable medium for positive psychosocial interventions. Evidence supports that long-term psychosocial adjustment of cancer survivors is enhanced by increased social support, particularly from other survivors [33]. Besides using ethnodrama as an effective knowledge translation strategy, we noted that breast cancer survivors' involvement in the creation of the ethnodrama resulted in strong bonding among the participants with a positive psycho-social effect [34].

The ethnodrama performance of the lived experiences of breast cancer survivors, along with the performing participants' interviews have been posted on YouTube (see the link in the method section). Readers are encouraged to watch the video for a better understanding of the ethnodrama. In order to assess enduring effect of ethnodrama on breast cancer survivors and health care professionals' knowledge and attitudes we interviewed a subgroup of members of the audiences (health care professionals and survivors with arm morbidity) at 4 and 8 months post-performance. The results will be reported separately.

\section{Conclusions}

The current study extends the application of ethnodrama as a knowledge translation strategy to the domain of breast cancer-induced arm morbidity To our knowledge, this is a first study that has used ethnodrama as a channel to convey findings related to the arm problems associated with breast cancer survivorship. Our study revealed a positive effect on the attitudes of breast cancer survivors and health care professionals, suggesting this is a promising avenue for further cancer survivorship research and knowledge translation.

\section{Acknowledgements}

The authors wish to thank all breast cancer survivors for their participation in this study and the Canadian Institutes for Health Research - Regional
Partnership Program for their generous support of the reported research.

\section{Funding Source}

This study is the part of a project funded by the Canadian Institutes for Health Research (grant \#000781-000).

\section{Competing Interests}

The authors have declared that no competing interest exists.

\section{References}

1. Hack TF, Kwan WB, Thomas-Maclean RL, et al. Predictors of arm morbidity following breast cancer surgery. Psychooncology. 2010;19:1205-12.

2. Paskett ED, Stark NN: Lymphedema: Knowledge, treatment, and impact among breast cancer survivors. Breast J 2000:6:373-378.

3. Donker M, Rutgers E, van de Velde C, et al: Axillary lymph node dissection vs axillary radiotherapy: A detailed analysis of morbidity. ASBS Annual Meeting. Presented April 30, 2014.

4. Lucci A, McCall LM, Beitsch PD, et al: Surgical complications associated with sentinel lymph node dissection (SLND) compared with SLND alone in the American College of Surgeons Oncology Group Trial Z0011. J Clin Oncol 2007:24:3657-3663.

5. McLaughlin SA, Wright MJ, Morris KT et al. Prevalence of Lymphedema in Women With Breast Cancer 5 Years After Sentinel Lymph Node Biopsy or Axillary Dissection: Objective Measurements. J Clin Oncol 2010;26:5213-5219.

6. Wilke LG, McCall LM, Posther KE, et al. Surgical complications associated with sentinel lymph node biopsy: Results from a prospective international cooperative group trial. Ann Surg Oncol 2006;13:491-500.

7. Langer I, Guller U, Berclaz G, et al: Morbidity of sentinel lymph node biopsy (SLN) alone versus SLN and completion axillary lymph node dissection after breast cancer surgery: A prospective Swiss multicenter study on 659 patients. Ann Surg 2007;245:452-461.

8. Engel J, Kerr J, Schlesinger-Raab A, et al: Axilla surgery severely affects quality of life: Results of a 5-year prospective study in breast cancer patients. Breast Cancer Res Treat 2003;79:47-57.

9. Ahmed RL, Prizment A, Lazovich D, et al: Lymphedema and quality of life in breast cancer survivors: The Iowa Women's Health Study. J Clin Oncol. 2008;26:5689-96.

10. Fu MR. Breast cancer-related lymphedema: Symptoms, diagnosis, risk reduction, and management. World J Clin Oncol. 2014;5:241-7.

11. Hack TF, Cohen L, Katz J, et al. Physical and psychological morbidity after axillary lymph node dissection for breast cancer. J Clin Oncol 1999;17:143-149.

12. Thomas-MacLean R, Spriggs P, Quinlan E, et al. Arm Morbidity and Disability: Reporting the Current Status from Canada. J Lymphoedema 2010;5:33-38.

13. Quinlan E, Thomas-MacLean R, Hack TF, et al. The Impact of Breast Cancer among Canadian Women: Disability and Productivity. Work 2009;34:285-96.

14. Quinlan E, Thomas-MacLean R, Hack TF, et al. Breast Cancer Survivorship and Work Disability. J Disabil Policy Stud. 2011;22:18-27.

15. Thomas- MacLean R, Towers A, Quinlan E, et al. "This is a kind of betrayal": A Qualitative Study of Disability after Breast Cancer. Curr Oncol 2009;16: 26-32.

16. Quinlan, E., Thomas, R., Hack, T., et al. Lymphedema Trajectories Among Breast Cancer Survivors. J Lympheodema 2014;9:12-19.

17. Paskett ED. Breast cancer-related lymphedema: attention to a significant problem resulting from cancer diagnosis. J Clin Oncol 2008;26:5666.

18. Kwan ML, Shen L, Munneke JR, et al. Patient awareness and knowledge of breast cancer-related lymphedema in a large, integrated health care delivery system. Breast Cancer Res Treat. 2012;135:591-602.

19. Shaw RM, Thomas R. Role of GPs in breast cancer-related arm morbidity care. J Lymphoedema; 2011;6:10-19.

20. Shaw RM, Thomas R. The information needs and media preferences of Canadian cancer specialists regarding breast cancer treatment related arm morbidity. Eur J Cancer Care. 2014;23:98-110.

21. Gray R., Fitch M., Labrecque M., \& Greenberg M. Reactions of health professionals to a research-based theatre production. J Cancer Educ 2003;18: 223-229.

22. Kontos, P., Poland, B. Mapping new theoretical and methodological terrain for knowledge translation: Contributions from critical realism and the arts. Implement Sci 2009;4:1-10.

23. Rossiter, K., Kontos, P., Colantonio, A., et al. Staging data: Theatre as a tool for analysis and knowledge transfer in health research. Soc Sci Med 2008;66:130-146.

24. Rieger K, Schultz AS. Exploring Arts-Based Knowledge Translation: Sharing Research Findings Through Performing the Patterns, Rehearsing the Results, Staging the Synthesis. Worldviews Evid Based Nurs. 2014;11:133-9.

25. [Internet] Breast Cancer Survivors Ethnodrama. http://www.breastcancersurvivors-ethnodrama.ca/survivors_resources.php 
26. Hill DA, Horick NK, Isaacs C, et al. Long-term risk of medical conditions associated with breast cancer treatment. Breast Cancer Res Treat. 2014;145:233-43.

27. Eccles SA, Aboagye EO, Ali S, et al. Critical research gaps and translational priorities for the successful prevention and treatment of breast cancer. Breast Cancer Res. 2013;15:R92.

28. Boissel, J-P. \& E. Amsallem. Bridging the gap between therapeutic research results and physician prescribing decisions: knowledge transfer, a prerequisite to knowledge translation. Eur J Clin Pharmacol 2004;60:609-616.

29. Davis, D. Continuing education, guideline implementation, and the emerging transdisciplinary field of knowledge translation. J Contin Educ Health Prof. 2006;26:5-12.

30. Holmes B, Scarrow G, Schellenberg M. Translating evidence into practice: the role of health research funders. Implement Sci. 2012;7:39.

31. Landry R, Amara N, Lamari M. Utilization of social science research knowledge in Canada. Research Policy 2001;30:333-349.

32. McLean RK, Graham ID, Bosompra K, et al. Understanding the performance and impact of public knowledge translation funding interventions: protocol for an evaluation of Canadian Institutes of Health Research knowledge translation funding programs. Implement Sci. 2012;7:57.

33. Taylor S, Falke R, Shoptaw J, Lichtman R. Social Support, Support Groups, and the Cancer Patient. J Consul Clin Psychol 1996;54:608-615.

34. Quinlan, E. Thomas, R., Ahmed, S., et al. The Aesthetic Rationality of the Popular Expressive Arts: Lifeworld Communication Among Breast Cancer Survivors Living with Lymphedema. Soc Theory Health 2014;12:291-312. 